\title{
LIFETIME AND INTERGENERATIONAL FITNESS CONSEQUENCES OF HARMFUL MALE INTERACTIONS FOR FEMALE LIZARDS
}

\author{
J.-F. Le Galliard, ${ }^{1,2,4}$ J. Cote, ${ }^{1}$ and P. S. Fitze ${ }^{1,3}$ \\ ${ }^{1}$ Laboratoire Fonctionnement et Evolution des Systèmes Ecologiques, CNRS UMR 7625, Université Pierre et Marie Curie, Case 237, \\ 7 Quai St. Bernard, 75005 Paris, France \\ ${ }^{2}$ Centre for Ecological and Evolutionary Synthesis, University of Oslo, P.O. Box 1050, Blindern, Oslo NO-0316 Norway \\ ${ }^{3}$ Museo Nacional de Ciencias Naturales (MNCN-CSIC), Calle José Gutierrez Abascal 2, 28006 Madrid, Spain
}

\begin{abstract}
Male mating behaviors harmful to females have been described in a wide range of species. However, the direct and indirect fitness consequences of harmful male behaviors have been rarely quantified for females and their offspring, especially for long-lived organisms under natural conditions. Here, lifetime and intergenerational consequences of harmful male interactions were investigated in female common lizards (Lacerta vivipara) using field experiments. We exposed females to male harm by changing the population sex ratio from a normal female-biased to an experimental male-biased sex ratio during the first experimental year. Thereafter, females and their first generation of offspring were monitored during two additional years in a common garden with a female-biased sex ratio. We found strong immediate fitness costs and lower lifetime reproductive success in females subjected to increased male exposure. The immediate fitness costs were partly mitigated by direct compensatory responses after exposure to male excess, but not by indirect benefits through offspring growth, offspring survival, or mating success of offspring. These results support recent empirical findings showing that the direct costs of mating are not outweighed by indirect benefits.
\end{abstract}

Key words: direct costs; fitness compensation; indirect benefits; multiple mating; sexual conflict; sexual selection.

\section{INTRODUCTION}

In females, mating interactions with males range from obvious mutualism to severe antagonism (Andersson 1994, Arnquist and Rowe 2005). Males may provide females with direct benefits, such as parental care, or with indirect benefits, for example good genes for parasite resistance (Andersson 1994). On the other hand, males can use coercive strategies to gain mating advantages, like sexual harassment or infanticide, thus promoting a sexual conflict over mating (Clutton-Brock and Parker 1995, Chapman et al. 2003). Indeed, behavioral studies spanning a broad range of species suggest that males often harm females during mating (reviewed by Arnqvist and Rowe 2005). For example, females can suffer significant costs due to harmful mating attempts by males, such as higher energy expenditure (e.g., Rowe et al. 1994), reduced fecundity from exposure to toxic male seminal fluids (e.g., Chapman et al. 1995), or lower survival caused by traumatic inseminations (e.g., Crudington and SivaJothy 2000).

Empirical data supporting the existence of a sexual conflict over mating have accumulated, but Orteiza et al. (2005) emphasized that these data suffer from two main

Manuscript received 13 December 2006; revised 10 May 2007; accepted 1 June 2007. Corresponding Editor: A. Sih.

${ }^{4}$ E-mail: galliard@biologie.ens.fr drawbacks. First, the net costs of male behavior have been rarely quantified throughout the lifetime of females. Lifetime costs of harmful male mating behaviors are well documented only in a few laboratory systems (e.g., Chapman et al. 1995, Martin and Hosken 2003, Linder and Rice 2005), but similar studies are exceedingly rare for longer-lived organisms and/or in natural conditions (see Maklakov et al. [2005] and references therein). The costs and benefits of mating may be affected by laboratory conditions where, e.g., population density and food availability are different from the conditions in the wild (Hosken and Tregenza 2006). Second, whether females can counterbalance the direct costs of mating by indirect benefits is still debated (e.g., Cordero and Eberhard 2003, Pizarri and Snook 2003, Arnqvist and Rowe 2005). For example, Cameron et al. (2003) showed that indirect benefits could play a role in the maintenance of apparently harmful male mating behaviors when females obtain "good genes" that increase the average survival or sexual competitiveness of their offspring (e.g., Kokko 2001). The relative magnitude of the direct costs and indirect benefits for females has been quantified in laboratory populations of fruit flies and house crickets (Head et al. 2005, Orteiza et al. 2005, Stewart et al. 2005, Rice et al. 2006). These studies yielded contradictory results, suggesting that measures of intergenerational effects in a larger number of systems and in more natural conditions are needed (Hosken and Tregenza 2006). Here, we quantify lifetime 
and intergenerational fitness consequences of harmful male mating behaviors for female common lizards (Lacerta vivipara Jacquin 1787) under near field conditions.

We manipulated male aggression (persistent courtship, mating attempts, and copulation itself) by increasing exposure to males for females during one year using an adult sex ratio manipulation. This sex ratio manipulation affected the number of potential partners for females and therefore the opportunity for a sexual conflict during mating (see Methods). We reported previously that the survival and fecundity of female lizards plummeted as a consequence of sexual aggression by males during mating (Fitze et al. 2005, Le Galliard et al. 2005b). However, these studies did not unravel whether females may compensate or even outweigh the mating costs via indirect benefits through offspring quality. Female common lizards may gain indirect benefits from more multiple mating, through increased genetic diversity of their clutch, and/or through "good genes" for the viability of their offspring (Laloi et al. 2004, Fitze et al. 2005, Richard et al. 2005). It is also unclear whether the current costs of mating were amplified by long-lasting deleterious effects on females, or, on the contrary, compensated for by positive responses in the future. For example, females may lower their reproductive investment in response to increased male aggression and reallocate the saved energy into future reproduction (Reyer et al. 1999). Here, we tested for indirect benefits through offspring quality and for possible trade-offs between successive reproductive events by monitoring females and their first generation of offspring during two additional years in a common garden with a female-biased sex ratio. We investigate the consequences of the sex ratio manipulation on the lifetime reproductive success of females, including immediate and delayed responses of sexual conflict. We also study the body growth, survival, and mating success of the first generation of offspring in order to quantify the intergenerational effects of the sex ratio manipulation.

\section{Material And Methods}

\section{Study species}

Lacerta vivipara is a small ground-dwelling ovoviparous lizard species in which sexes share overlapping home ranges (Lecomte et al. 1994). Natural populations include three main life stages: juveniles, yearlings ( 1 year old), and adults ( $\geq 2$ years old [Massot et al. 1992]). In our study area, some, but not all, females start reproduction at the age of one year. Mating occurs during April and May each year. The mating behavior involves antagonistic elements since the male chases and bites the female on the flanks. Parturitions start from the beginning of June until the end of July and females lay on average five transparent, soft-shelled eggs (range 112). Offspring hatch shortly after parturition and are autonomous thereafter.

\section{Experimental design}

We maintained 12 experimental populations in enclosures located in a natural meadow at the Ecological Research Station of Foljuif (France, $60 \mathrm{~m}$ above sea level, $48^{\circ} 17^{\prime} \mathrm{N}, 2^{\circ} 41^{\prime} \mathrm{E}$ ). The enclosure's habitat matched the species' natural habitat and each enclosure $(10 \times 10 \mathrm{~m})$ was surrounded by plastic walls to prevent lizards from escaping and covered by a net. The enclosures were thus protected from all avian and terrestrial predators (see Boudjemadi et al. [1999a] for more details). Lizards introduced in these populations at the start of the study (June 2002) were obtained from natural populations of the Cévennes area $\left(44^{\circ} 30^{\prime} \mathrm{N}\right.$, $3^{\circ} 45^{\prime}$ E, $1400-1600 \mathrm{~m}$ above sea level) and marked by toe clipping.

A detailed description of the experimental procedures has been given elsewhere (Fitze et al. 2005, Le Galliard et al. 2005b) and we summarize our study design here (see also Fig. 1). To enhance exposure to males, we created six populations with a male-biased adult sex ratio and compared the fate of females from these populations with that of females from six populations with a female-biased adult sex ratio (Fig. 1). Yearling ( $n$ $=12$ per population) and juvenile $(n=42-45$ per population) sex ratios were held constant and balanced (1:1) in all populations. The adult sex ratio $(n=18$ adults per population) of the female-biased populations (22\% of males) corresponds to the mean adult sex ratio in the natural source habitat (average adult sex ratio $=18 \% \pm$ $0.18 \%$ [mean $\pm \mathrm{SD}])$. The adult sex ratio of male-biased populations (78\% of males) corresponds to the extremes in the same natural habitat (Le Galliard et al. 2005a).

We have previously reported on the number of mating attempts for all females that survived the first year of this experiment (Fitze et al. 2005). We counted the number of mating scars on the belly of the females and assessed multiple-partner mating by determining paternity of all fertilized eggs using microsatellite genotyping. Our analyses demonstrated (1) that female lizards were subjected to an overall increase in the number of mating attempts by males in male-biased populations (3.62 vs. 1.49 per female [Le Galliard et al. 2005b]), (2) that this effect was stronger in females that had multiply sired clutches than in monandrous females (Fitze et al. 2005), and (3) that male-biased sex ratios increased the number of multiply sired clutches for polyandrous females (Fitze et al. 2005). Altogether, these results indicate that mating rate was higher in the male-biased populations than in the female-biased populations.

Adult sex ratios vary significantly through time and space in natural populations (Le Galliard et al. 2005a), with male-biased sex ratios ( $>60 \%$ adult males) occurring in $\sim 10 \%$ of the social neighborhoods inhabited by female lizards. After one year of sex ratio manipulation, we released all alive females $(n=148)$ and their offspring $(n=551)$ together with other lizards from all age and sex classes into new populations with a female-biased sex ratio (Fig. 1). The female-biased sex ratio was chosen to 

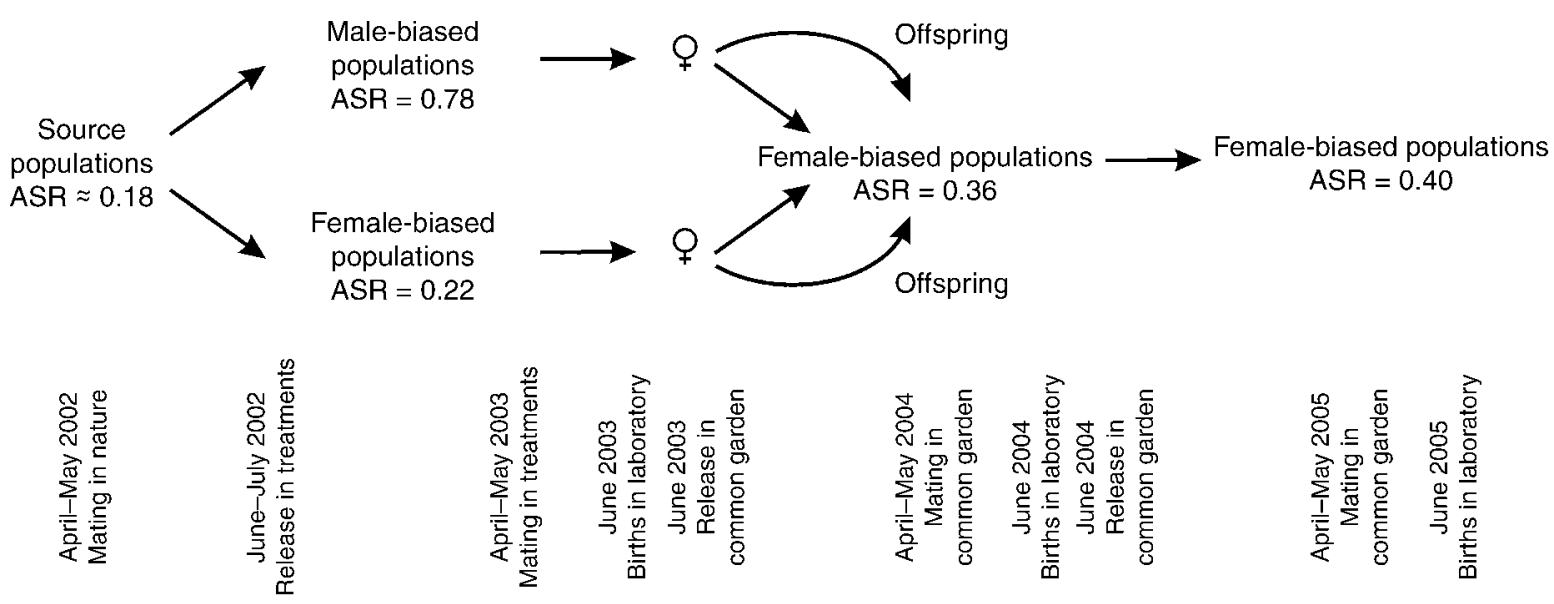

Field experiment Lab maintenance Field experiment Lab maintenance Field experiment Lab maintenance

FIG. 1. Experimental design. The opportunity for sexual conflict over mating was manipulated by establishing populations with female- or male-biased (male excess) adult population sex ratios (ASR). After one year, females and offspring from both treatments were released in female-biased populations where they were maintained during two additional years. In the first year, 18 adult females, 10 adult males, six yearling males, and six yearling females were introduced in each of eight enclosures. In the second year, nine adult females, six adult males, eight yearling males, and eight yearling females were introduced in each of 16 enclosures. Lifetime and intergenerational fitness consequences were scored by measuring various fitness components for females and their first generation of offspring during each year of the study.

mimic the long-term mean sex ratio experienced by female lizards in the wild. We released these other lizards together with the experimental groups to guarantee similar population structures among enclosures and years. Within each enclosure, the offspring sex ratio was held constant $(1: 1)$ and the proportion of offspring originating from male-biased and female-biased populations was similar. The number of released lizards per age class and the sex ratio per age class were also held constant between populations. We monitored females and their first generation offspring during two additional years following the sex ratio manipulation by recapturing lizards in each enclosure during June of each year (Fig. 1). The lizards released in the enclosures that had not participated in the sex ratio experiment were not included in the analysis.

\section{Monitoring procedures}

For all lizards, snout-vent length (SVL) was measured to the nearest millimeter before release and for each capture during the three experimental years. Each June, we captured all female lizards and housed them in individual cages in the laboratory (Fig. 1). Females were classified as gravid or non-gravid by palpation of the abdominal cavity and their cages were then checked daily for freshly laid or hatched eggs at 09:00 and 14:00 hours. Approximately one hour after completion of hatching, mothers were removed from their cages, and the number of unhatched eggs, dead offspring, and viable offspring was counted. Mothers were released in outdoor enclosures a few days following parturition. This monitoring design allowed measuring the most important lifetime fitness components (body growth, survival, and reproductive outputs) for all females released in the experiment until the end of the study period (Fig. 1). We retained three reproductive characteristics for our analysis, namely proportion of gravid females, clutch success (proportion of viable offspring per clutch), and fecundity (number of viable offspring). During the first year of the study, body mass loss during parturition was also calculated to evaluate maternal investment per egg.

For the offspring generation born in the laboratory after the first year of the sex ratio manipulation (Fig. 1), we measured SVL and body mass (to the nearest milligram) and released lizards a few days after hatching. A small part of the tip of the tail of each hatchling was collected for future paternity assignment. For the rest of the experiment, we captured all lizards in June of each year, measured SVL, and kept females in the laboratory to assess pregnancy and reproductive output as described previously. Furthermore, we collected genetic tissue in all offspring born from the monitored enclosures for future paternity assignment. The DNA of offspring and all putative fathers was extracted and five to six microsatellite loci were amplified as described in Boudjemadi et al. (1999b) to infer paternity. Paternity of all offspring was unambiguously attributed to the potential fathers, including sons from the first generation of offspring (see Fitze et al. [2005] for details on assignment tests). The design thus allowed us to measure the most relevant fitness components (offspring size and condition at birth, growth, survival to maturity, and mating success of both females and males) for all offspring born from the experimental populations during their two first years of 
life (Fig. 1). Body condition was defined as body mass adjusted for body length by including SVL as a covariate in the statistical analysis. Body mass and body length were not log-transformed prior to the linear regression because the relationship was linear on the natural scale in the body length interval considered here and the residuals were normally distributed.

\section{Statistical analysis}

For the analysis of female traits, all models contained the following factors: adult sex ratio treatment and initial age class as fixed effects, SVL of the female as a covariate, and enclosure nested within treatment as a random factor. For the age classification, we separated females in three age categories according to their age at the start of the study (juvenile, year-born; yearling, $1 \mathrm{yr}$ old; or adult, $\geq 2$ yr old). However, since our study lasted for three years, we refer to true age rather than initial age category when we present results on age classification. For example, the juvenile cohort was born in 2002, was one year old in 2003, two years old in 2004, and three years old in 2005. For the analysis of the offspring traits, the model also included a random effect of family nested within enclosure and sex ratio treatment. Initial models contained all factors and model selection was done backward. Normally distributed variables were analyzed with linear models using the MIXED procedure in SAS version 8.2 (Littell et al. 1996). The assumptions of these models (normality and homogeneous variance of residuals) were fulfilled. Binomial data were modeled with logistic regression using the GLIMMIX procedure with a logit link (Littell et al. 1996). The goodness of fit of logistic regressions was checked with a Pearson chisquare test (McCullagh and Nelder 1989).

The ability to detect meaningful compensatory responses in females and indirect effects in offspring was estimated by conducting post hoc power analyses for pairwise comparisons between male-biased and female-biased populations after the sex ratio manipulation. Significant treatment effects observed during the first year of the study showed a range of 1.7-fold to fivefold reduction in fitness components of females from male-biased populations (Table 1). We therefore asked whether we had power to detect effects of the same order of magnitude after the sex ratio manipulation. Precisely, we calculated the power $(1-\beta)$ associated with a doubling in fitness components of females and offspring from male-biased populations relative to females from male-biased populations. Power was calculated with a two-tailed type I risk $(\alpha)$ of 0.05 and with the variance estimates and sample sizes of our study (Quinn and Keough 2002).

\section{RESULTS}

\section{Lifetime fitness effects of the sex ratio manipulation}

We quantified the total reproductive success (TRS) of each female released in the experiment as follows:

$$
\begin{aligned}
\mathrm{TRS}= & S(2002 \rightarrow 2003)\left\{\mathrm{FE}_{2003}+S(2003 \rightarrow 2004)\right. \\
& \left.\times\left[\mathrm{FE}_{2004}+S(2004 \rightarrow 2005) \times \mathrm{FE}_{2005}\right]\right\}
\end{aligned}
$$

where $S$ stands for annual survival probability and FE stands for annual fecundity (number of viable offspring produced each year). The TRS was affected by the sex ratio treatment, the initial age class, and the interaction between sex ratio treatment and initial age class (treatment $F_{1,10}=25.37, P=0.0005$; age $F_{2,425}=$ 19.84, $P<0.0001$; treatment $\times$ age $F_{2,425}=13.26, P<$ $0.0001, n=441)$. Females aged at the start of the study as yearlings and adults had lower TRS in male-biased populations than in female-biased populations (Tukey contrasts, all $P<0.0001$ ), but no treatment effect was found for females aged as juveniles at the start of the study (Tukey contrast, $P=0.66$; Fig. 2A).

The effects of the sex ratio manipulation on each fitness component present in Eq. 1 and on a number of additional fitness components are summarized in Table 1. During the first study year, i.e., during the sex ratio treatment, the mortality of yearling and adult females was dramatically increased by male excess, while the survival of juvenile females was not significantly diminished (see Le Galliard et al. 2005b). At the end of the first year, the proportion of unfertilized eggs was small and similar between treatments (12 out of 753 eggs screened for genetic paternity [Fitze et al. 2005]) and the clutch success (proportion of viable offspring) was also not affected by the sex ratio manipulation (treatment $F_{1,10}=0.48, P=0.50 ;$ SVL $F_{1,111}=19.62, P<0.0001$; logit slope $=0.19 \pm 0.07[$ mean $\pm \mathrm{SE}]$ ). However, in male-biased populations, gravid females produced fewer offspring than in female-biased populations (Poisson regression, treatment $F_{1,10}=8.44, P=0.02$; SVL $F_{1,111}=$ 56.5, $P<0.0001$; Table 1). Furthermore, oviposition date was affected by a significant interaction between treatment and age $\left(F_{2,107}=4.08, P=0.02\right.$, Table 1$)$. In male-biased populations, the older females oviposited on average $10.38 \pm 2.96$ days (mean \pm SE) later than in female-biased populations (individual contrasts, $P=$ $0.0007)$, while the treatment had no effect on oviposition date in two-year-old (individual contrasts, $0.88 \pm 2.59$ days, $P=0.73$ ) and one-year-old females (individual contrasts, $0.69 \pm 2.06$ days, $P=0.74$ ).

We analyzed the body growth, survival, and reproductive characteristics of females after the sex ratio manipulation (Table 1). Two positive delayed responses were observed in females from male-biased populations during the first year following the sex ratio manipulation. First, the annual survival probability of females was higher for females from male-biased populations $\left(F_{1,10}=\right.$ 6.87, $P=0.03, n=148$, Table 1). Second, clutch success, i.e., the proportion of viable eggs per clutch, was higher for females from male-biased populations than from female-biased populations $\left(F_{1,10}=7.15, P=0.02, n=71\right.$, Table 1). The sex ratio treatment had no detectable effects on body growth and fecundity. No delayed effects in any of the studied life history traits were found in the 
TABLE 1. Effects of the adult sex ratio manipulation for female common lizards on lifetime and intergenerational fitness components.

\begin{tabular}{|c|c|c|c|c|c|c|c|c|}
\hline \multirow[b]{2}{*}{ Study stage } & \multirow[b]{2}{*}{ Component } & \multirow[b]{2}{*}{$\begin{array}{l}\text { Age } \\
\text { class }\end{array}$} & \multicolumn{2}{|c|}{$\begin{array}{l}\text { Male-biased } \\
\text { populations }\end{array}$} & \multicolumn{2}{|c|}{$\begin{array}{c}\text { Female-biased } \\
\text { populations }\end{array}$} & \multirow[b]{2}{*}{$P$} & \multirow[b]{2}{*}{$\begin{array}{l}\text { Statistical } \\
\text { power }\end{array}$} \\
\hline & & & Mean & $\overline{\text { size }}$ & Mean & $\underset{\text { size }}{\text { Sample }}$ & & \\
\hline \multicolumn{9}{|l|}{$\begin{array}{l}\text { Lifetime fitness } \\
\text { component }\end{array}$} \\
\hline \multirow[t]{3}{*}{ First year } & survival & Adult & $0.20[0.08,0.43]$ & 24 & $0.69[0.54,0.80]$ & 84 & $<0.001$ & \\
\hline & & Yearling & $0.16[0.07,0.34]$ & 36 & $0.79[0.59,0.90]$ & 36 & $<0.001$ & \\
\hline & & Juvenile & $0.17[0.10,0.28]$ & 132 & $0.29[0.19,0.42]$ & 134 & 0.10 & \\
\hline \multirow[t]{6}{*}{ End of first year } & proportion gravid & $\geq 2$ years & $0.91[0.55,0.98]$ & 11 & $0.98[0.92,0.99]$ & 85 & 0.15 & \\
\hline & & 1 year & $0.65[0.44,0.82]$ & 23 & $0.56[0.40,0.71]$ & 39 & 0.52 & \\
\hline & fecundity & & $2.48[1.68,3.65]$ & 22 & $4.27[3.75,4.86]$ & 102 & 0.02 & \\
\hline & oviposition date $\dagger$ & $\geq 3$ years & $29.99 \pm 2.93$ & 4 & $19.61 \pm 1.16$ & 54 & 0.007 & \\
\hline & & 2 years & $19.54 \pm 2.35$ & 6 & $18.65 \pm 1.09$ & 27 & 0.73 & \\
\hline & & 1 year & $27.02 \pm 2.23$ & 12 & $26.32 \pm 1.91$ & 21 & 0.74 & \\
\hline \multirow[t]{2}{*}{ Second year } & survival & & $0.70[0.49,0.85]$ & 30 & $0.42[0.32,0.53]$ & 118 & 0.03 & 0.99 \\
\hline & body growth $(\mathrm{mm})$ & & $2.25 \pm 0.47$ & 21 & $2.49 \pm 0.30$ & 50 & 0.69 & 0.69 \\
\hline \multirow[t]{2}{*}{ End of second year } & fecundity & & $2.58 \pm 0.61$ & 21 & $2.22 \pm 0.41$ & 50 & 0.64 & 0.88 \\
\hline & clutch success & & $0.89[0.59,0.98]$ & 21 & $0.49[0.29,0.69]$ & 50 & 0.02 & 0.99 \\
\hline \multirow[t]{2}{*}{ Third year } & survival & & $0.35[0.20,0.54]$ & 21 & $0.36[0.36,0.61]$ & 50 & 0.95 & 0.73 \\
\hline & body growth $(\mathrm{mm})$ & & $4.06 \pm 0.98$ & 7 & $2.09 \pm 0.70$ & 17 & 0.09 & 0.32 \\
\hline End of third year & fecundity: & & $6.42 \pm 1.06$ & 7 & $6.06 \pm 0.68$ & 17 & 0.77 & 0.99 \\
\hline \multicolumn{9}{|l|}{$\begin{array}{l}\text { Intergenerational } \\
\text { fitness components }\end{array}$} \\
\hline \multirow{2}{*}{ Hatching } & body size & & $22.27 \pm 0.31$ & 43 & $22.55 \pm 0.20$ & 508 & 0.42 & 1 \\
\hline & body condition (mg) & & $188.46 \pm 0.31$ & 43 & $194.28 \pm 5.62$ & 508 & 0.41 & 1 \\
\hline $\begin{array}{l}\text { From hatching to } \\
\text { two years old }\end{array}$ & survival & & $0.14[0.06,0.31]$ & 43 & $0.12[0.09,0.16]$ & 508 & 0.69 & 0.52 \\
\hline $\begin{array}{l}\text { From hatching to } \\
\text { one year old }\end{array}$ & size growth $(\mathrm{mm})$ & & $28.52 \pm 1.30$ & 12 & $28.40 \pm 0.48$ & 139 & 0.93 & 1 \\
\hline \multirow{2}{*}{$\begin{array}{l}\text { From one to two } \\
\text { years old }\end{array}$} & daughters' fecundity & & $1.50 \pm 1.15$ & 6 & $1.55 \pm 0.22$ & 82 & 0.96 & 0.28 \\
\hline & sons' mating success & & $1.16 \pm 1.17$ & 6 & $2.67 \pm 0.60$ & 57 & 0.39 & 0.29 \\
\hline
\end{tabular}

Notes: Results are predicted means ( \pm SE or $95 \% \mathrm{CL})$ of the statistical models reported in Results: Lifetime fitness effects of the sex ratio manipulation and Intergenerational fitness effects of the sex ratio manipulation. Results are given for each age class when the age effect was significant, and the $P$ value of a $t$ test for individual contrasts is indicated. Fecundity was measured as the total number of viable offspring per clutch, including non-gravid females in the calculation. Clutch success was given by the proportion of viable offspring per clutch among gravid females. Body size was measured by snout-vent length. Body condition was measured by body mass, to the nearest milligram, after controlling for body size. Daughters' fecundity was given by the total number of viable offspring produced by each daughter during her two first years of life, excluding daughters that died at the juvenile stage. Sons' mating success was given by the total number of viable offspring produced by each son during the two first years of life, excluding sons that died at the juvenile stage. The statistical power is reported for pairwise comparisons between male-biased and female-biased populations after the sex ratio manipulation. For details on model selection, statistics, and power analysis, see Materials and methods: Statistical analysis.

$\dagger$ Days from June 1 .

$\$$ Non-gravid females were included in this calculation.

second year after the sex ratio manipulation (Table 1). We lacked some power to detect meaningful compensatory responses through body growth, but the power was satisfactory for survival and fecundity data (Table 1).

To test whether the detected fitness compensations could balance the current fecundity costs of male excess, we calculated reproductive success conditional on survival during the first year of the experiment [i.e., for females where $S(2002 \rightarrow 2003)=1$ in Eq. 1]. We found no difference in reproductive success between treatments among those females (treatment $F_{1,10}=0.10, P=0.76$; age $F_{2,144}=12.17, P<0.0001$; treatment $\times$ age $F_{2,142}=$ $0.55, P=0.57 ; n=158$, Fig. $2 \mathrm{~B})$. This indicates that fecundity costs of male mating attempts during the first year of the experiment were compensated by fitness benefits later in life.

\section{Intergenerational fitness effects of the sex ratio manipulation}

None of the fitness components of the first generation of offspring was found to differ between sex ratio treatments (Table 1). First, females of both sex ratio treatments invested similar mass per unit egg in malebiased and female-biased populations (Fig. 3). Second, offspring SVL and offspring body condition at hatching were similar for mothers from male-biased and femalebiased populations (Table 1). Although clutch size and hatching date differed between sex ratio treatments and although offspring were smaller and leaner when born from larger clutches or later in the season, the absence of a sex ratio effect on offspring traits remained when clutch size and hatching date were controlled for in the model (Table 2). Offspring survival, offspring body 

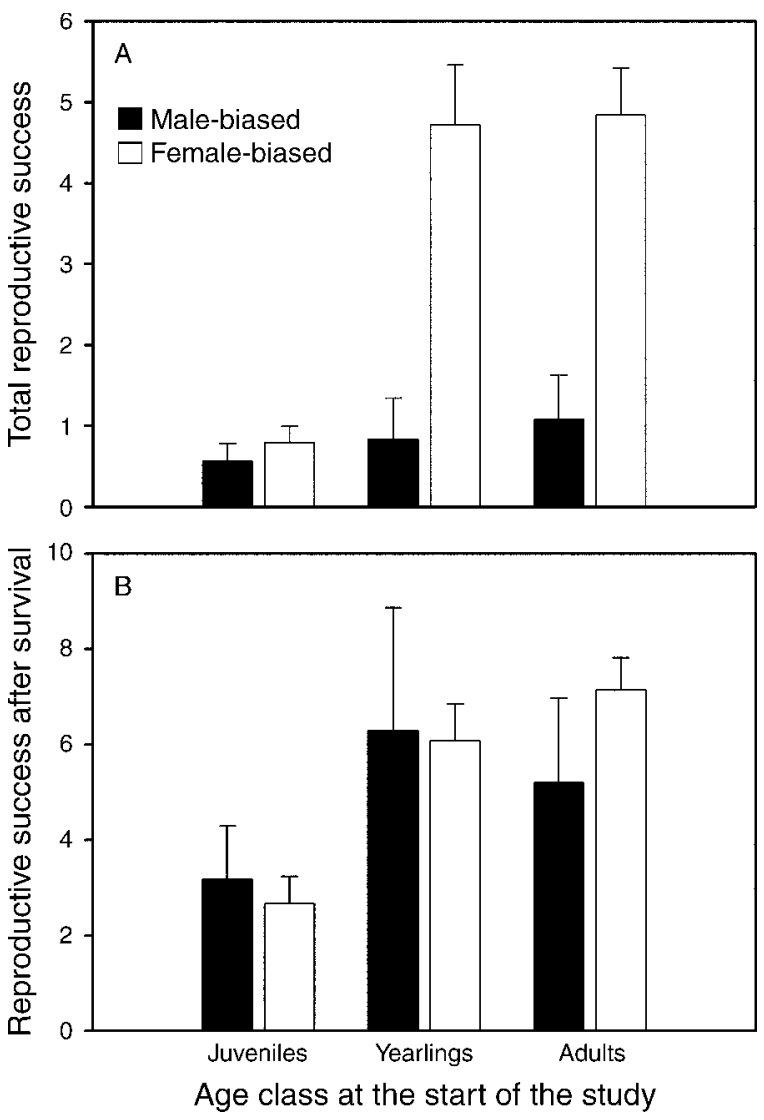

FIG. 2. Lifetime fitness consequences of the sex ratio manipulation. (A) Total reproductive success of female common lizards (mean + SE) per sex ratio treatment and age class at the start of the study. The total reproductive success was measured by counting the number of viable offspring produced by each female during the three years of the study. (B) Reproductive success of female common lizards conditional on their survival during the first year of the experiment (mean + SE) per sex ratio treatment and age class at the start of the study. Comparison with panel A shows that the main lifetime costs were the direct negative effects of males on female survival during the first year of the experiment.

growth, and the mating success of both sons and daughters were not affected by the maternal sex ratio treatment (Table 1). Further, there was also no sex ratio effect when applying separate analyses of growth and survival at the juvenile ( $n=551$ released individuals) and at the yearling stage $(n=150$ released individuals, all $P$ $>0.51)$. We lacked some power to detect meaningful changes in the mating success of sons and daughters, but the power was satisfactory for pairwise comparisons with other fitness components (Table 1).

\section{Discussion}

Earlier, we reported that male-biased sex ratios increased the female's mating rate in the common lizard Lacerta vivipara (Fitze et al. 2005). Furthermore, compared to a female-biased sex ratio, a male-biased sex ratio decreased female survival and fecundity (Le
Galliard et al. 2005b). Here, we quantified the lifetime and intergenerational fitness consequences of sex ratio manipulation by monitoring females and their first generation of offspring during two additional years in a common garden with a female-biased sex ratio. A total of 24 of the 441 released females $(\sim 5 \%)$ and 68 of the 551 first-generation offspring $(\sim 12 \%)$ survived until the end of the study, showing that our measurements of female and offspring fitness are close to lifetime reproductive success. The field experiment yielded three main results. First, we found unambiguous evidence for lower lifetime reproductive success in females of malebiased populations. Second, females surviving after they had been exposed to male excess were able to compensate the fecundity costs caused by males by surviving and breeding better in the year following the sex ratio manipulation. Yet, these compensatory responses were not strong enough to outweigh the survival costs incurred during the sex ratio manipulation. This shows that the main lifetime costs of the sexual conflict were the direct negative effects of males on female survival. Third, the direct lifetime costs of male excess were not mitigated by indirect benefits through viability, growth or mating success of offspring.

\section{Lifetime effects}

In a previous study, we reported that the excess of adult males had immediate survival and fecundity costs for female lizards (Le Galliard et al. 2005b). Here, we additionally show that adult females of male-biased populations oviposited later in the season, which may reduce the time for growth and maturation of their

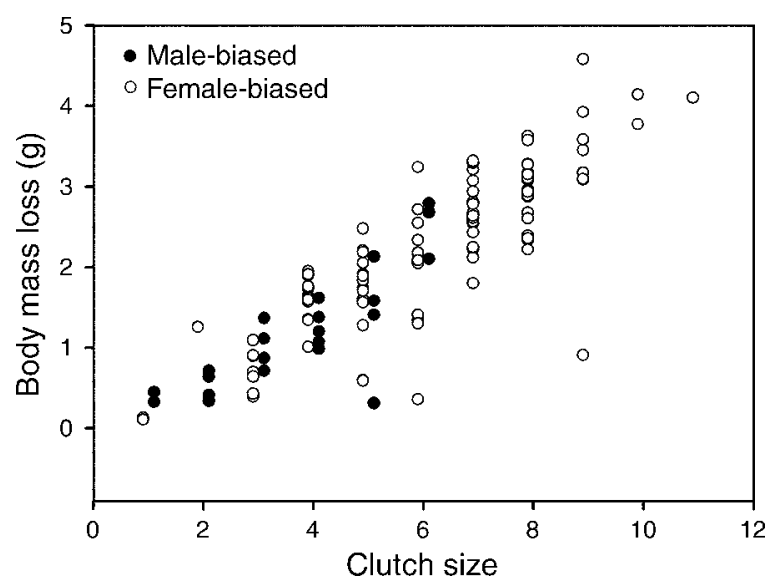

FIG. 3. Body mass loss during parturition in relation to total clutch size in females from male-biased and female-biased populations. The body mass loss increased linearly with clutch size $\left(F_{1,107}=83.25, P<0.0001\right)$ but did not differ between sex ratio treatments $\left(F_{1,10}=0.01, P=0.92\right)$. Body mass loss controlled for clutch size also increased with snout-vent length, SVL $\left(F_{1,107}=8.44, P=0.0045\right)$ and decreased with date of parturition $\left(F_{1,107}=12.09, P=0.0007\right)$. Male-biased and female-biased populations were examined within a similar range of clutch size values; symbols are staggered only for purposes of presentation. 
TABLE 2. Effects of sex ratio manipulation on offspring snout-vent length (SVL) and condition (body mass controlled for SVL) when controlling for mother size, clutch size, and hatching date.

\begin{tabular}{lrrrrr}
\hline \hline \multirow{2}{*}{\multicolumn{1}{c}{ Factor }} & \multicolumn{2}{c}{ SVL $(\mathrm{mm})$} & & \multicolumn{2}{c}{ Body mass $(\mathrm{mg})$} \\
\cline { 2 - 3 } \cline { 5 - 6 } & \multicolumn{1}{c}{ Estimate } & Test statistic & & Estimate & Test statistic \\
\hline Intercept & $21.97 \pm 0.31$ & & & $203.20 \pm 5.52$ & \\
Treatment (F) & $0.22 \pm 0.35$ & $F_{1,10}=0.40$ & & $-3.63 \pm 6.14$ & $F_{1,10}=0.35$ \\
Mother SVL & $0.11 \pm 0.03$ & $F_{1,498}=16.36^{* * * *}$ & & $1.84 \pm 0.51$ & $F_{1,497}=12.94^{* *}$ \\
Clutch size & $-0.18 \pm 0.07$ & $F_{1,498}=6.68^{*}$ & & $-3.99 \pm 1.31$ & $F_{1,497}=9.24^{* *}$ \\
Hatching date & $-0.07 \pm 0.01$ & $F_{1,498}=19.46^{* * *}$ & & $-0.80 \pm 0.28$ & $F_{1,497}=8.22^{* *}$ \\
SVL at hatching & & & $9.87 \pm 0.64$ & $F_{1,497}=240.49^{* * *}$ \\
Enclosure(Treatment) & $0.13 \pm 0.10$ & $Z=1.33$ & & $35.64 \pm 33.35$ & $Z=1.07$ \\
Clutch(Enclosure, Treatment) & $0.70 \pm 0.12$ & $Z=5.89^{* * *}$ & & $246.06 \pm 41.25$ & $Z=5.96^{* * *}$ \\
\hline
\end{tabular}

Notes: Estimates $( \pm \mathrm{SE})$ are given for female-biased populations $(\mathrm{F})$ and with centered covariates. Significance was determined after correction for multiple tests using a sequential Bonferroni procedure. Larger females produced longer and heavier offspring; offspring SVL and body condition decreased with increasing clutch size; and hatching date was negatively correlated with offspring size and condition.

$* P<0.05 ; * * P<0.01 ; * * P<0.001$

offspring (Olsson and Shine 1997, Sinervo 1999). The negative impact of male excess on female survival and reproduction could be the outcome of intersexual competition and/or male aggression during mating, but our observations provided strong evidence that male aggression, and thus sexual conflict, was more important than intersexual competition (details can be found in Le Galliard et al. 2005b). The number of mating scars and wounds on the females due to males' biting during mating attempts were more frequent in male-biased populations than in female-biased populations, indicating stronger male harassment in male-biased populations. Furthermore, the treatment affected female mortality during mating, but not before the mating season, which supports the sexual conflict hypothesis. Against the intersexual competition hypothesis, female body growth was not affected by the manipulation and the pre-hibernation body condition of females did not differ between treatments. Given that females grow and accumulate their body reserves mostly during summer and autumn (Massot et al. 1992), these results suggest only weak competitive asymmetry between sexes and strong male aggression during the mating season. Several nonexclusive mechanisms of sexual conflicts might be involved and can not be distinguished with our field data. Proximate physiological and behavioral processes could include direct injuries causing death or pathogen infection (Shine et al. 2001), harassment during mating constraining critical foraging and basking activities (Magurran and Seghers 1994), and/or chronic stress during mating that suppressed reproduction or immune defenses in females (Svensson et al. 2001).

Fitness costs in females were age dependent. First, survival and total reproductive success were negatively affected by male excess in yearlings and adults, but not significantly different in juveniles. Second, oviposition dates of females were affected by male excess only in adult females. These results are somewhat surprising given that older females have a larger body size and should potentially be more resistant to sexual aggression than younger females (Clutton-Brock and Parker 1995). Age-dependent costs in female lizards could be explained by the fact that older females emerge earlier from wintering than younger females (Bauwens and Verheyen 1985), by age-assortative mating between male and female lizards (Richard et al. 2005), and/or by male mating preferences for older females. As a consequence, the adult sex ratio manipulation may have increased the duration and/or the intensity of male aggression disproportionately in older females.

The lifetime fitness effect of male excess on females was caused primarily by immediate survival costs. Indeed, the immediate fecundity costs were compensated by positive fitness responses later in female life (Fig. 2B). These compensatory responses could be explained by pronounced survival selection in male-biased populations leading to the survival of higher quality females. However, no differences in survival selection were found between male- and female-biased populations in body size and body condition during the first year of the experiment (Le Galliard et al. 2005a), indicating that differences in survival selection may not explain the observed compensatory responses. Another alternative explanation is that females traded-off current with future reproduction. Such life history responses may be a very general feature of sexual conflicts in iteroparous species, which may enable females to mitigate some of the direct costs of mating (Reyer et al. 1999). However, the evolutionary impact of these counter-responses may be limited if females lack the ability to avoid social environments where the risks of harmful mating are high (Rowe et al. 1994).

\section{Intergenerational effects}

Although the mating behavior of males caused direct costs for females, female lizards might benefit from mating if large indirect benefits counterbalance the direct lifetime costs (e.g., Kokko 2001, Cameron et al. 2003, Cordero and Eberhard 2003). We have shown elsewhere that higher exposure to males increased the 
number of male mating attempts and the numbers of multiply sired clutches for polyandrous females (Fitze et al. 2005). Uller and Olsson (2005) suggested that more multiple mating could be a strategy to avoid sperm limitation or bet-hedge against genetic incompatibilities, since sperm may be a limiting resource in this species. However, we found no evidence for such indirect benefits. The manipulation had no detectable effects on proportion of gravid females, proportion of fertilized eggs per clutch and proportion of viable eggs per clutch. This strongly suggests that the number and quality of sperm was not limiting in our study. More multiple mating might enhance other indirect benefits for female common lizards, e.g., through a higher genetic diversity of their clutch, bet-hedging against genetic defects in offspring, and/or "good genes" for their offspring (see Laloi et al. [2004], Fitze et al. [2005], and Richard et al. [2005] for discussion of these potential benefits of multiple mating in this species). However, against this hypothesis, mothers did not invest more resources per egg in male-biased populations. Furthermore, mean offspring traits at hatching (size and condition) did not differ between treatments. There were also no differences between sex ratio treatments in the other components of offspring fitness investigated here, i.e., body growth, survival, and mating success during the two first years of life. The absence of treatment effects on mean offspring traits at hatching and body growth held despite a strong statistical power (see Table 1). The power to detect meaningful differences in offspring survival was not entirely satisfactory, but our raw data suggested no trend toward a better survival for offspring from malebiased populations. Thus, we are confident that we did not miss an important compensatory effect through offspring viability.

We lacked power to assess potential benefits of mating via reproduction in daughters and via sexual selection on sons (the "sexy son" hypothesis [Weatherhead and Robertson 1979]). This loss of power was the consequence of strong viability selection during the two first years of life and substantial variance in male mating success. Several authors have claimed that large indirect benefits via sons' attractiveness or sexual competitiveness may often outweigh potential direct costs of mating (e.g., Kokko 2001, Cordero and Eberhard 2003, Pizarri and Snook 2004). Others have found little empirical support for large indirect benefits through sons (Arnqvist and Rowe 2005 and references therein). In a study on female crickets, however, Head et al. (2005) reported a two-fold increase in the sexual attractiveness of sons derived from mating with preferred males, which appeared to compensate for the direct costs of mating. This sexy son effect was due to mating rate, male size, or other male properties. Although our sample size was somewhat limited, there was no support for large indirect benefits through sons' mating success in our study. On the contrary, the mating success of sons from male-biased populations was half that of sons from female-biased populations (see Table 1). Thus, we are also confident that indirect benefits via sexy son effects could not compensate for the direct costs of mating in our study.

\section{Conclusion}

Relatively few studies have been able to assess the wide range of potential fitness consequences of harmful mating attempts for females, especially for long-lived organisms under field conditions (Andersson 1994, Maklakov et al. 2005, Hosken and Tregenza 2006). Our field experiment shows that harmful male mating importantly affected lifetime female fitness through immediate survival costs. These direct costs could not be compensated by indirect benefits through offspring viability or mating success. The absence of counteradaptations in female common lizards may be the outcome of an evolutionary arm race where males win the conflict, e.g., because fitness rewards of aggression for males are larger than fitness benefits of counteradaptations for females (Clutton-Brock and Parker 1995). Furthermore, selection for female counter-adaptations in natural populations of common lizards may be inefficient since average sex ratios are female biased and male-biased populations are rapidly declining (Le Galliard et al. 2005b). A similar pattern was found by Orteiza et al. (2005) who show that the direct lifetime fitness costs of harmful male interactions for females were seven times larger than the indirect fitness benefits in laboratory populations of the fruit fly (see also Stewart et al. 2005, Rice et al. 2006). This suggests that male mating behavior may often impose serious costs on females that cannot be compensated for entirely.

\section{ACKNOWLEDGMENTS}

We thank E. Svensson for thorough critical comments on the manuscript, and D. Mersch and M.-L. Jarzat for field assistance. Funds were received from the French Ministry of Education and Research (ACI "Jeunes Chercheurs 2001"), the French Ministry of Environment (ACI "Invasions biologiques"), the European Research Training Network "ModLife" (HPRN-CT-2000-00051), the Federal Office of Education and Science (BBW No. 01.0254 to P. S. Fitze), and the Swiss National Science Foundation (No. PBBEA-104428 to P. S. Fitze).

\section{Literature Cited}

Andersson, M. 1994. Sexual selection. Princeton University Press, Princeton, New Jersey, USA.

Arnqvist, G., and L. Rowe. 2005. Sexual conflict. Princeton University Press, Princeton, New Jersey, USA.

Bauwens, D., and R. F. Verheyen. 1985. The timing of reproduction in the lizards Lacerta vivipara: differences between individual females. Journal of Herpetology 19:353364.

Boudjemadi, K., J. Lecomte, and J. Clobert. 1999a. Influence of connectivity on demography and dispersal in two contrasting habitats: an experimental approach. Journal of Animal Ecology 68:1207-1224.

Boudjemadi, K., O. Martin, J. C. Simon, and A. Estoup. 1999 b. Development and cross-species comparison of microsatellite markers in two lizard species, Lacerta vivipara and Podarcis muralis. Molecular Ecology 8:518-520. 
Cameron, E. T., T. Day, and L. Rowe. 2003. Sexual conflict and indirect benefits. Journal of Evolutionary Biology 16: 1055-1060.

Chapman, T., G. Arnqvist, J. Bangham, and L. Rowe. 2003. Sexual conflict. Trends in Ecology and Evolution 18:41-47.

Chapman, T., L. F. Liddle, J. M. Kalb, M. F. Wolfner, and L. Partridge. 1995. Cost of mating in Drosophila melanogaster females is mediated by male accessory gland products. Nature 373:241-244.

Clutton-Brock, T. H., and G. A. Parker. 1995. Sexual coercion in animal societies. Animal Behaviour 49:1345-1365.

Cordero, C., and W. G. Eberhard. 2003. Female choice of sexually antagonistic male adaptations: a crucial review of some current research. Journal of Evolutionary Biology 16: $1-6$.

Crudington, H. S., and M. Siva-Jothy. 2000. Genital damage, kicking and early death. Nature 407:855-856.

Fitze, P. F., J.-F. Le Galliard, P. Federici, M. Richard, and J. Clobert. 2005. Conflict over multiple partner mating among males and females of polygynandrous common lizards. Evolution 59:2451-2459.

Head, M. L., J. Hunt, M. D. Jennions, and R. Brooks. 2005. The indirect benefits of mating with attractive males outweigh the direct costs. PLOS Biology 3:289-294.

Hosken, D. J., and T. Tregenza. 2006. Do bad husbands make good fathers? Current Biology 15:R836-R838.

Kokko, H. 2001. Fisherian and "good genes" benefits of mate choice: how (not) to distinguish between them. Ecology Letters 4:322-326.

Laloi, D., M. Richard, J. Lecomte, M. Massot, and J. Clobert. 2004. Multiple paternity in clutches of common lizard Lacerta vivipara: data from microsatellite markers. Molecular Ecology 13:719-723.

Lecomte, J., J. Clobert, M. Massot, and R. Barbault. 1994. Spatial and behavioural consequences of a density manipulation in the common lizard. Ecoscience 1:300-310.

Le Galliard, J.-F., P. Fitze, J. Cote, M. Massot, and J. Clobert. 2005a. Female common lizards (Lacerta vivipara) do not adjust their sex-biased investment in relation to the adult sex ratio. Journal of Evolutionary Biology 18:1455-1463.

Le Galliard, J.-F., P. Fitze, R. Ferrière, and J. Clobert. $2005 b$. Sex ratio bias, male aggression, and population collapse in lizards. Proceedings of the National Academy of Sciences (USA) 102:18231-18236.

Linder, J. E., and W. R. Rice. 2005. Natural selection and genetic variation for female resistance to harm from males. Journal of Evolutionary Biology 18:568-575.

Littell, R. C., G. A. Millinken, W. W. Stroup, and R. D. Wolfinger. 1996. SAS systems for mixed models. SAS Institute, Cary, North Carolina, USA.

Magurran, A. E., and B. H. Seghers. 1994. A cost of sexual harassment in the guppy, Poecilia reticulata. Proceedings of the Royal Society of London B 258:89-92.

Maklakov, A. A., T. Bilde, and Y. Lubin. 2005. Sexual conflict in the wild: elevated mating rate reduces female lifetime reproductive success. American Naturalist 165:S38-S45.

Martin, O. Y., and D. J. Hosken. 2003. Costs and benefits of evolving under experimentally enforced polyandry or monogamy. Evolution 57:2765-2772.
Massot, M., J. Clobert, T. Pilorge, J. Lecomte, and R. Barbault. 1992. Density dependence in the common lizard: demographic consequences of a density manipulation. Ecology 73:1742-1756.

McCullagh, P., and J. A. Nelder. 1989. Generalized linear models. Second edition. Chapman and Hall, London, UK.

Olsson, M., and R. Shine. 1997. The seasonal timing of oviposition in sand lizards (Lacerta agilis): why early clutches are better. Journal of Evolutionary Biology 10:369-381.

Orteiza, N., J. E. Linder, and W. R. Rice. 2005. Sexy sons from re-mating do not recoup the direct costs of harmful male interactions in the Drosophila melanogaster laboratory model system. Journal of Evolutionary Biology 18:1315-1323.

Pizarri, T., and R. R. Snook. 2003. Sexual conflict and sexual selection: chasing away paradigm shifts. Evolution 57:12231236.

Pizarri, T., and R. R. Snook. 2004. Sexual conflict and sexual selection: measuring antagonistic coevolution. Evolution 58: 1389-1393.

Quinn, G. P., and M. J. Keough. 2002. Experimental design and data analysis for biologists. Cambridge University Press, Cambridge, UK.

Reyer, H. U., G. Frei, and C. Som. 1999. Cryptic female choice: frogs reduce clutch size when amplexed by undesired males. Proceedings of the Royal Society of London B 266:21012107.

Rice, W. D., A. D. Stewart, E. H. Morrow, J. E. Lindler, N. Orteiza, and P. G. Byrne. 2006. Assessing sexual conflict in the Drosophila melanogaster laboratory model system. Philosophical Transactions of the Royal Society of London B 361:287-299.

Richard, M., J. Lecomte, M. de Fraipont, and J. Clobert. 2005. Age-specific mating strategies and reproductive senescence. Molecular Ecology 14:3147-3155.

Rowe, L., G. Arnqvist, A. Sih, and J. Krupa. 1994. Sexual conflict and the evolutionary ecology of mating patternswater striders as a model system. Trends in Ecology and Evolution 9:289-293.

Shine, R., P. LeMaster, I. T. Moore, M. M. Olsson, and R. T. Mason. 2001. Bumpus in the snake den: effects of sex, size, and body condition on mortality of red-sized garter snakes. Evolution 55:598-604.

Sinervo, B. 1999. Mechanistic analysis of natural selection and a refinement of Lack's and William's principles. American Naturalist 154:S26-S42.

Stewart, A. D., E. H. Morrow, and W. R. Rice. 2005. Assessing putative interlocus sexual conflict in Drosophila melanogaster using experimental evolution. Proceedings of the Royal Society of London B 272:2029-2036.

Svensson, E., B. Sinervo, and T. Commendant. 2001. Densitydependent competition and selection on immune function in genetic lizard morphs. Proceedings of the National Academy of Sciences (USA) 98:12561-12565.

Uller, T., and M. Olsson. 2005. Multiple copulations in natural populations of lizards: evidence for the fertility assurance hypothesis. Behaviour 142:45-56.

Weatherhead, P. J., and R. J. Robertson. 1979. Offspring quality and the polygyny threshold: "sexy son" hypothesis. American Naturalist 113:201-208. 